\title{
Lipid profile in Polish women following lacto-ovo-vegetarian and vegan diets - preliminary study
}

\author{
Patrycja Gogga', Aleksandra Śliwińska², Ewa Aleksandrowicz-Wrona² and \\ Sylwia Małgorzewicz ${ }^{2}$ \\ 'Department of Commodity Science, Medical University of Gdańsk, Gdańsk, Poland; 2Department of Clinical Nutrition, Medical University of \\ Gdańsk, Gdańsk, Poland
}

\begin{abstract}
The European Atherosclerosis Society and American College of Cardiology and American Heart Association guidelines have introduced several new recommendations, according to which vegetarian diets are concerned as beneficial for the cardiovascular system. This study was aimed to investigate the lipid profile in $\mathbf{5 6}$ healthy Polish young women - 13 vegans, 18 lacto-ovo-vegetarians and 25 omnivores. HDL cholesterol concentrations were higher in the vegans and vegetarians than in the omnivores. LDL cholesterol levels were lower in the vegans than in both other diet groups. The use of a vegetarian - especially vegan - diet in Polish conditions is associated with a favorable lipid profile and may have cardioprotective effects.
\end{abstract}

Key words: diet, veganism, vegetarianism, lipid profile, cholesterol Received: 11 April, 2021; revised: 22 June, 2021; accepted: 23 July, 2021; available on-line: 06 October, 2021

$\square$ e-mail: sylwiam@gumed.edu.pl

Acknowledgements of Financial Support: The study was a part of the Nutritional Status of Vegetarians Research, financed by Medical University of Gdańsk (grant no: MN 01-0098/08).

Abbreviations: CVD, cardiovascular disease; BMI, body mass index; DL, law density lipoprotein HDL, high density lipoprotein; MUFA, mono-unsaturated fatty acids; PUFA, poly-unsaturated fatty acids SFA, saturated fatty acids; TG, triglycerides

\section{INTRODUCTION}

The World Health Organization reports that in 2016 CVD was the leading cause of death in Poland, responsible for $46 \%$ of deaths from noncommunicable diseases (WHO, 2020). It is commonly known that diet is a crucial factor affecting cardiovascular health (Mozarrafian et al., 2016). Nowadays, more and more people adopt dietary patterns based on plant foods (Kamiński et al., 2010). It is worth noting that - if composed properly - such diets meet all nutritional requirements according to the Academy of Nutrition and Dietetics (Melina et al., 2016). Moreover, they appear to be beneficial in the prevention and treatment of certain diseases like diabetes type 2, obesity, and various afflictions of the cardiovascular system (Oussalah et al., 2016). Indeed, the European Atherosclerosis Society, and the American College of Cardiology, and the American Heart Association guidelines have introduced several new suggestions and Mediterranean and vegetarian diets are examples of diet patterns that are currently being deliberated upon, as to whether they are healthy for the cardiovascular system (Arnett et al., 2019; Wojda et al., 2020).
Inter alia, hypercholesterolemia, and hypertriglyceridemia are considered risk factors in the development of CVD, and the lipidogram is the most frequently performed test in primary care, even in the population of healthy people. To the best of our knowledge, there is no research comparing lipid profiles in vegans, vegetarians, and omnivores in Poland; thus, this study was aimed to assess the plasma levels of total cholesterol, low density lipoprotein (LDL) cholesterol, high density lipoprotein (HDL) cholesterol, and triglycerides (TG) in healthy women from three diet groups - lacto-ovovegetarian (hereafter referred to as the vegetarian group), vegan, and omnivore - in order to evaluate the influence a meat-free diet may have on the lipid profile and, as a consequence, on the cardiovascular risk.

\section{MATERIALS AND METHODS}

The study involved 56 healthy women - 13 vegans (median age 30.0 (3.5) years), 18 lacto-ovo-vegetarians (29.5 (4.0) years), and 25 omnivores (24.0 (1.0) years). All participants had normal Body Mass Index (BMI), i.e. 18.5-24.99. Participants of the study group were recruited into the study via electronic media. The study was approved by the local Independent Bioethics Commission for Research (NKBBN/508/2012).

The inclusion criterion (for the non-omnivores) was refraining from eating meat for at least one year continuously for both (vegetarian/vegan) study groups and from consuming any other animal-derived products (i.e. dairy, eggs, honey) for the vegan group. Additional criteria were the absence of any chronic diseases and eating disorders, and the absence of pregnancy or lactation.

The control (omnivore) group was recruited from among employees and students of the Medical University of Gdańsk. The additional inclusion criteria were similar; however, subjects from the control group consumed meat regularly. The basic characteristics of the study groups are presented in Table 1.

Table 1. Comparison of basic characteristics in different diet groups. Data are shown as median \pm quartile deviation.

\begin{tabular}{lllll}
\hline Variables & $\begin{array}{l}\text { Vegan } \\
(\mathrm{n}=13)\end{array}$ & $\begin{array}{l}\text { Vegetarian } \\
(\mathrm{n}=18)\end{array}$ & $\begin{array}{l}\text { Omnivore } \\
(\mathrm{n}=25)\end{array}$ & $p$ value \\
\hline Age & $30.0 \pm 3.5$ & $29.5 \pm 4.0$ & $24.0 \pm 1.0$ & $<0.01$ \\
\hdashline BMI & $20.0 \pm 1.4$ & $20.6 \pm 1.4$ & $20.7 \pm 1.5$ & 0.62 \\
\hdashline Body fat (\%) & $18.9 \pm 1.8$ & $22.2 \pm 3.8$ & $24.7 \pm 3.0$ & 0.21 \\
\hline
\end{tabular}


Table 2. Food sources of principal macronutrients in different diet groups. The order od products reflects the frequency of intake.

\begin{tabular}{|c|c|c|c|}
\hline \multirow{2}{*}{ Diet type } & \multicolumn{3}{|c|}{ Food sources of macronutrients } \\
\hline & protein & carbohydrates & fat \\
\hline vegan & $\begin{array}{l}\text { pulses (variuos), tofu, soy milk, nuts, } \\
\text { seeds }\end{array}$ & $\begin{array}{l}\text { wholemeal bread, oats, groats, rice, } \\
\text { potatoes, pulses, confectionery }\end{array}$ & $\begin{array}{l}\text { avocado, nuts (various), seeds (flax- } \\
\text { seed, seasame), oils (olive, flaxseed, } \\
\text { rapeseed) }\end{array}$ \\
\hline vegetarian & $\begin{array}{l}\text { dairy (yogurth, milk, cheese, cottage } \\
\text { cheese), pulses (various), soy milk, } \\
\text { tofu, eggs, nuts }\end{array}$ & $\begin{array}{l}\text { wholemeal bread, oats, groats, rice, } \\
\text { potatoes, pulses, confectionery }\end{array}$ & $\begin{array}{l}\text { butter, oils (olive, rapeseed), avocado, } \\
\text { nuts (various), eggs }\end{array}$ \\
\hline omnivore & $\begin{array}{l}\text { dairy (cheese, yogurth, milk, cottage } \\
\text { cheese), meat (chicken, pork), eggs, } \\
\text { fish }\end{array}$ & $\begin{array}{l}\text { white bread, oats, confectionery, } \\
\text { groats, rice, potatoes, pasta }\end{array}$ & butter, mayonnaise, nuts (almonds) \\
\hline
\end{tabular}

\section{Diet assessment}

Prior to the onset of the study, all the included women provided informed consent. They had a consultation with a well-trained, experienced interviewer during which they performed a 24-hour diet recall in order to estimate their nutrient intake. To avoid potential modifications of eating habits, respondents were not informed about the dietary assessment method before the interview. Respondents had the opportunity to use an album of photographs of popular food products and meals, showing portion sizes for corresponding food quantities; this made assessing of the portion sizes easier and more reliable. None of the participants was using any diet supplements during, and prior to, the onset of the study. The data collected from the interviews was used to estimate the intake of energy and selected diet components, which was calculated using Cronometer ${ }^{\circledR}$ (https:// cronometer.com/). During consultation respondents also described their feeding habits; this information allowed for the assessment of food sources of basic macronutrients, which are presented in Table 2.

\section{Anthropometry}

Body mass was measured by the electronic scale (Tanita TBF-400A, Japan) and body fat content was measured using the bioelectrical impedance method with Bodystat 1500 (UK). The consultant also measured the height of the participants, which was essential for calculating the BMI.

\section{Laboratory parameters}

The blood was collected (after an overnight fast) by a nurse. It was immediately centrifuged; the obtained plasma was stored at $-80^{\circ} \mathrm{C}$. Plasma concentrations of HDL and LDL total cholesterol, and triglycerides were examined directly using enzymatic colorimetric tests (Co-

Table 3. Serum levels of lipid fractions in different diet groups. After exclusion of outliers values, data were analysed using one-way ANOVA $(*)$ or Kruskal-Wallis test.

\begin{tabular}{|c|c|c|c|c|c|c|}
\hline Variables & Diet type & $\mathrm{Me} \pm \mathrm{QD}$ & Min & Max & $p$ value & $\mathrm{n}$ \\
\hline \multirow{3}{*}{$\mathrm{TG}[\mathrm{mg} / \mathrm{dl}]$} & vegan & $67.0 \pm 12.5$ & 43.0 & 82.0 & \multirow{3}{*}{0.42} & \multirow{3}{*}{54} \\
\hline & vegetarian & $70.0 \pm 20.5$ & 40.0 & 153.0 & & \\
\hline & omnivore & $66.0 \pm 18.0$ & 46.0 & 233.0 & & \\
\hline \multirow{3}{*}{ Total cholesterol $[\mathrm{mg} / \mathrm{dl}]^{*}$} & vegan & $126.0 \pm 18.0$ & 104.0 & 189.0 & $p 1=0.09$ & \multirow{3}{*}{56} \\
\hline & vegetarian & $168.5 \pm 21.0$ & 117.0 & 207.0 & $p 2=0.004$ & \\
\hline & omnivore & $150.0 \pm 16.0$ & 116.0 & 205.0 & $p 3=0.23$ & \\
\hline \multirow{3}{*}{$\mathrm{LDL}[\mathrm{mg} / \mathrm{dl}]^{*}$} & vegan & $61.0 \pm 18.0$ & 43.0 & 107.0 & $p 1=0.04$ & \multirow{3}{*}{55} \\
\hline & vegetarian & $90.5 \pm 9.5$ & 52.0 & 118.0 & $p 2=0.02$ & \\
\hline & omnivore & $84.0 \pm 18.5$ & 44.0 & 148.0 & $p 3=0.88$ & \\
\hline \multirow{3}{*}{$\mathrm{HDL}[\mathrm{mg} / \mathrm{dl}]$} & vegan & $61.2 \pm 1.3$ & 56.6 & 66.2 & $p 1=0.001$ & \multirow{3}{*}{51} \\
\hline & vegetarian & $63.0 \pm 1.6$ & 56.0 & 66.8 & $p 2=0.82$ & \\
\hline & omnivore & $57.1 \pm 1.1$ & 47.7 & 68.9 & $p 3<0.001$ & \\
\hline
\end{tabular}

Me, median; QD, quartile deviation; Min, minimum value; Max, maximum value. Post-hoc analysis: $p 1$, vegan vs. omnivores; $p 2$, vegetarian vs. vegan; $p 3$, omnivore vs. vegetarian. 
Table 4. Daily intake of selected nutrients (shown as median \pm quartile deviation). After exclusion of outliers values, data were analysed using one-way ANOVA $(*)$ or Kruskal-Wallis test.

\begin{tabular}{|c|c|c|c|c|c|}
\hline \multirow{2}{*}{ Diet component } & \multicolumn{3}{|l|}{ Daily intake } & \multirow{2}{*}{$p$ value } & \multirow{2}{*}{$\mathrm{n}$} \\
\hline & Vegans & Vegetarians & Omnivores & & \\
\hline Lipids [g] & $49.15 \pm 38.30$ & $64.00 \pm 13.50$ & $55.55 \pm 11.42$ & 0.46 & 51 \\
\hline \multirow{3}{*}{ Proteins [g/kg b.w.] ${ }^{*}$} & \multirow{3}{*}{$0.78 \pm 0.27$} & \multirow{3}{*}{$0.81 \pm 0.22$} & \multirow{3}{*}{$1.23 \pm 0.31$} & $p 1=0.003$ & \multirow{3}{*}{53} \\
\hline & & & & $p 2=0.98$ & \\
\hline & & & & $p<0.001$ & \\
\hline Carbohydrates [g] & $276.55 \pm 51.62$ & $221.50 \pm 62.00$ & $232.60 \pm 35.10$ & 0.14 & 51 \\
\hline \multirow{3}{*}{ Fiber $[g]^{*}$} & \multirow{3}{*}{$50.90 \pm 7.18$} & \multirow{3}{*}{$32.50 \pm 8.50$} & \multirow{3}{*}{$24.90 \pm 6.65$} & $p 1<0.001$ & \multirow{3}{*}{49} \\
\hline & & & & $p 2=0.01$ & \\
\hline & & & & $p 3=0.03$ & \\
\hline \multirow{3}{*}{ Cholesterol [mg] } & \multirow{3}{*}{$0.00 \pm 0.00$} & \multirow{3}{*}{$40.80 \pm 71.80$} & \multirow{3}{*}{$217.65 \pm 104.85$} & $p<0.001$ & \multirow{3}{*}{50} \\
\hline & & & & $p 2=0.049$ & \\
\hline & & & & $p 3=0.01$ & \\
\hline \multirow{3}{*}{ SFA $[g]$} & \multirow{3}{*}{$8.65 \pm 7.60$} & \multirow{3}{*}{$18.00 \pm 9.90$} & \multirow{3}{*}{$22.95 \pm 5.95$} & $p 1=0.01$ & \multirow{3}{*}{52} \\
\hline & & & & $p 2=0.17$ & \\
\hline & & & & $p 3=0.84$ & \\
\hline MUFA [g] & $16.50 \pm 19.82$ & $20.90 \pm 7.00$ & $18.25 \pm 3.95$ & 0.45 & 51 \\
\hline \multirow{3}{*}{ PUFA [g] } & \multirow{3}{*}{$20.60 \pm 7.42$} & \multirow{3}{*}{$14.50 \pm 3.60$} & \multirow{3}{*}{$8.80 \pm 2.10$} & $p 1<0.001$ & \multirow{3}{*}{50} \\
\hline & & & & $p 2=0.24$ & \\
\hline & & & & $p 3=0.01$ & \\
\hline
\end{tabular}

Me, median; QD, quartile deviation. Post-hoc analysis: $p 1$, vegan vs. omnivores; $p 2$, vegetarian vs. vegan; $p 3$, omnivore vs. vegetarian.

bas, Roche Diagnostic, Germany). These widely applied methods are based on several enzymatic reactions; the last one is catalyzed by peroxidase and gives pigment. The color intensity of the final product was determined photometrically with an automatic chemistry analyzer, Hitachi 704 (Roche Diagnostic, Germany).

\section{STATISTICAL ANALYSIS}

All statistical analyses were performed in Statistica 12 (Statsoft). Since the distributions of the most analyzed variables were not normal (based on the Shapiro-Wilk test), the Kruskal-Wallis test was used for comparison between different diets. Dunn's test was applied posthoc. For normally distributed variables a one-way ANOVA test was used, with the Tuckey test applied post-hoc. To examine if there is a relationship between cholesterol fractions and selected diet components, Spearman's rank correlation coefficient was used. Values were regarded as outliers based on visual assessment; for which boxplots were used, such values were excluded from further analysis. Results were regarded as statistically significant when $p \leq 0.05$.

\section{RESULTS}

There were statistically significant differences in total cholesterol, in both LDL and HDL cholesterol levels. HDL was higher in vegans and vegetarians than in omnivores $(\mathrm{H} 2,51=31.06, p<0.001)$. LDL levels were lower in vegans than in both other diet groups ( $\mathrm{F} 2,55=4.45$, $p=0.02$ ). Total cholesterol levels were higher in vegetarians compared to vegans, but there were no statistically significant differences between omnivores and both plant diet groups $(\mathrm{F} 2,56=4.72, p=0.01)$. There were no differences in TG levels $(\mathrm{H} 2,54=1.74, p=0.42)$ between the analyzed groups (Table 3 ).

Analysis of 24-h dietary recalls showed disparities in consumed diet components. Both vegans and vegetarians ate more polyunsaturated fatty acids (PUFA) $(\mathrm{H} 2,50=23.16, p<0.001)$ than the control group, while only vegans consumed less saturated fatty acids (SFA) (H2,52=8.95, $p=0.01$ ) compared to the control group. There were no differences in monounsaturated fatty acids (MUFA) $(\mathrm{H} 2,51=1.61, p=0.45)$ intake (Table 4).

Within PUFA, vegans consumed more both $\mathrm{n}-3$ $\left(\mathrm{H}_{2,50}=15.27, p=0.0003\right)$ and $\mathrm{n}-6\left(\mathrm{H}_{2,49}=18.14, p=0.0003\right)$ fatty acids than omnivores. There were no differences in the intake of these fatty acids between vegans and vegetarians $(p=0.16$ and $p=0.71$ respectively). Vegetarians ate more n-6 fatty acids than omnivores $(p=0.008)$, while we found no difference in $\mathrm{n}-3$ consumption between these groups $(p=0.18)$.

All the analyzed groups presented different cholesterol consumption $(\mathrm{H} 2,50=30.54, p<0.001)$. Medians of cholesterol intake were $0 \mathrm{mg} /$ day for vegans, $40.80 \mathrm{mg} /$ day for vegetarians, and $217.65 \mathrm{mg} /$ day for omnivores. Omnivores ate significantly more protein than both plant diet groups $(F 2,53=10.65, p<0.001)$. Additionally, all groups differed in fiber consumption (F2,49=17.21, $p<0.001$ ), which was the highest in vegans and the lowest in omnivores. There were no differences in to- 
tal lipid $(\mathrm{H} 2,51=1.53, p=0.46)$ and total carbohydrate $(\mathrm{H} 2,51=3.92, p=0.14)$ consumption (Table 4).

There was a weak but statistically significant positive relationship between LDL cholesterol levels and body mass (Rs=0.36, $p=0.007$ ), as well as BMI (Rs=0.29, $p=0.03$ ) and percentage body fat (Rs=0.39, $p=0.003$ ). HDL cholesterol concentrations were moderately negatively correlated with cholesterol intake $(\mathrm{Rs}=-0.44$, $p=0.002$ ), weakly positively correlated with fiber consumption (Rs $=0.33, p=0.02$ ) and moderately positively correlated with PUFA intake ( $\mathrm{Rs}=0.45, p=0.002)$.

\section{DISCUSSION}

We observed lower levels of LDL cholesterol in vegans compared to omnivores, which is in accordance with other studies (Kim et al., 2012; Elorinne et al., 2016). Positive impact of plant diets on LDL cholesterol was also observed after nutritional intervention in numerous studies; for instance, in male and female Ethiopians who were supposed to follow a vegan diet for 7 weeks. After a one-week washout they switched to the omnivorous diet for another 7 weeks. This short-term vegan diet contributed to lower LDL cholesterol levels in both sexes compared to the omnivorous diet (Mekonen et al., 2018). It is worth noting that there were no differences in BMI between the studied groups in our work. Additionally, in each group participants consumed a similar amount of lipids.

LDL cholesterol was significantly higher in vegetarians than in vegans, and so was the total cholesterol. This may be attributed to several factors. Firstly, lactoovo-vegetarians - in contrast to vegans - consume animal-derived products, such as dairy and eggs, which are natural sources of exogenous cholesterol. Indeed, all analyzed groups presented significantly different cholesterol consumption (Table 3). Secondly, we noted statistically lower consumption of dietary fiber in the vegetarian group than among vegans, while the latter had the highest intake of this component among all the analyzed diet groups (Table 3 ). It has been recognized that a high consumption of fiber-rich foods displays strong hypocholesterolemic effects, and its cardioprotective action was confirmed in meta-analysis, which revealed a significant dose-dependent relationship between fiber (both soluble and insoluble) intake and the incidence and mortality of coronary heart disease (Wu et al., 2015).

In our study, HDL cholesterol was slightly but significantly higher in both vegans and vegetarians compared to the omnivores. Most papers demonstrate levels of each fraction of cholesterol-bearing lipoproteins lower in vegans than in their meat-eating counterparts (Elorinne et al., 2016), though there are some articles showing no differences between diet groups in HDL cholesterol levels (Kim et al., 2012; Kuchta et al., 2016). In a recent study, Brazilian vegetarians had statistically significantly higher levels of HDL cholesterol than their meat-eating counterparts, although they also had lower body weight and BMI (De Mello Barros Pimentel et al., 2019). A possible explanation for the observed levels of HDL cholesterol may be the dietary composition of fatty acids. As summarized by Manuelli and others (Manuelli et al., 2017) n-3 PUFA seem to induce a small increase in HDL cholesterol levels and in our work the intake of these fatty acids was considerably higher both in vegans and vegetarians compared to controls. What is more, we observed a positive correlation between HDL cholester$\mathrm{ol}$ and PUFA consumption. It is commonly known that vegetarians, especially strict ones, consume more n-6 acids than $n-3$, and the latter ones are associated with better lipid profiles and cardiovascular health (Saunders et al., 2013). Indeed, the intake of n-6 fatty acids was higher in both vegans and vegetarians from our study. However, vegans also consumed significantly higher amounts of n-3 PUFA than omnivores. Presumably, they consumed $\alpha$-linolenic acid, a natural precursor of eicosapentaenoic and docosahexa enoic acids in the human body. Most participants from this group consumed flaxseed oil and nuts (walnuts), which are a good source of this fatty acid for vegans. On the other hand, omnivores consumed no flaxseed and only little amounts of nuts, and - more importantly - fish, which are the basic source of n-3 PUFA in a non-vegan diet. Unfortunately, we found no difference in n-3 PUFA intake between vegetarians and omnivores.

We observed a positive correlation between HDL cholesterol and fiber consumption, which positively affects HDL cholesterol concentrations (Zhou et al., 2015). Additionally, it has been recognized that a lower consumption of sugars and refined carbohydrates is associated with increased HDL cholesterol levels (Lee et al., 2014). In our work there were no differences in total carbohydrate consumption. As vegans and vegetarians consumed significantly more fiber than meat-consumers, and the overall carbohydrate intake was statistically the same, it suggests less highly-absorbed mono- and disaccharides, and starch in the diet, which may be an additional factor promoting higher HDL cholesterol levels. Lastly, there are some papers describing the positive effects of soy consumption on the lipid profile, namely lowering LDL cholesterol and increasing HDL cholesterol blood levels (Jassi et al., 2010; Chi et al., 2016). It is commonly known that the intake of this legume is considerably higher in non-meat-eaters than in meat-consumers (Rizzo \& Baroni, 2018), and - as opposed to omnivores - participants from both plant-based diet groups consumed soy products, which may be another explanation for the observed results.

We observed no differences in serum TG concentrations between the analyzed diet groups (Table 2). Generally, studies on serum TG levels are inconsistent. The review of over twenty controlled and randomized trials showed that adopting dietary patterns based mainly on plant products, but also eggs and dairy, is effective in lowering blood concentrations of TG and LDL cholesterol; the effectiveness of intervention was bigger for strict plant diets (Ferdowsian \& Barnard, 2009). However, in the study conducted by Elorinne and others (Elorinne et al., 2016) in Finnish subjects, there were no statistically significant differences between vegans and their meat-eating counterparts in TG levels. On the contrary, Chiu and colleagues (Chiu et al., 2015) in their cross-sectional study including lacto-ovo-vegetarians, lacto-vegetarians, and vegans, observed that all meat-avoiding diet groups had higher TG levels than meat-consumers. A recent meta-analysis of 30 observational studies and nearly 20 controlled trials showed that diets excluding meat contribute to lower levels of all cholesterol fractions (total, LDL, and HDL), but not to any changes in TG levels (Yokoyama et al., 2017). Altogether, one can make a conclusion that levels of TG are under the influence of specific (individual) diet and life-style factors, rather than solely a general feeding pattern, and this issue requires further research.

The advantage of our work is the presentation of results in a homogeneous group of vegetarians and vegans in the Pomeranian region. Although, we are aware that 
the following study has some limitations. First, the number of participants was relatively small, and second, we used 24-hour dietary recall for diet assessment.

\section{CONCLUSIONS}

We conclude that diets devoid of meat, especially a strict vegan diet, may positively affect the lipid profile by lowering LDL and increasing HDL cholesterol levels, without altering TG concentrations. These changes in blood cholesterol fractions may be partially explained by higher consumption of PUFA and fiber by subjects on both types of plant diets. The encouraging effects of plant-based diets with a high fiber and PUFA content should be part of individualized primary and secondary prevention of CVD. Enriching omnivore diets with these nutrients may also be beneficial for cardio-vascular health.

\section{REFERENCES}

Arnett DK, Blumenthal RS, Albert MA, Buroker AB, Goldberger ZD, Hahn EJ, Him-Melfarb CD, Khera A, Lloyd-Jones D, McEvoy JW, Michos ED, Miedema MD, Muñoz D, Smith SC Jr, Virani SS, Williams KA Sr, Yeboah J, Ziaeian B (2019) 2019 ACC/AHA Guideline on the primary prevention of cardiovascular disease a report of the American College of Cardiology/American Heart Association Task Force on Clinical Practice Guidelines. Circulation 140: e596e646. https://doi.org/10.1161/CIR.0000000000000678

Chi XX, Zhang T, Zhang DJ, Yu W, Wang QY, Zhen JL (2016) Effects of isoflavones on lipid and apolipoprotein levels in patients with type 2 diabetes in Heilongjiang Province in China. J Clin Biochem Nutr 59: 134-138. https://doi.org/10.3164/jcbn.15-147

Chiu YF, Hsu CC, Chiu TH, Lee CY, Liu TT, Tsao ChK, Chuang SCh, Hsiung ChA (2015) Cross-sectional and longitudinal comparisons of metabolic profiles between vegetarian and non-vegetarian subjects: a matched cohort study. Br J Nutr 114: 1313-1320. https://doi.org/10.1017/S0007114515002937

De Mello Barros Pimentel CV, Tucunduva Philippi S, Lazari Simomura V, Teodorov E (2019) Nutritional status, lifestyle and lipid profile in vegetarians. Int J Cardiol 32: 623-624. https://doi.org/10.5935/23594802.20190044

Elorinne AL, Alfthan G, Erlund I, Kivimäki H, Paju A, Salminen I, Turpeinen U, Voutilainen S, Laakso J (2016) Food and nutrient intake and nutritional status of Finnish vegans and non-vegetarians. PloS One 11: e0148235. https://doi.org/10.1371/journal. pone. 0148235

Ferdowsian HR, Barnard ND (2009) Effects of plant-based diets on plasma lipids. Am J Cardiol 104: 947-956. https://doi.org/10.1016/j. amjcard.2009.05.03

Jassi HK, Jain A, Arora S, Chitra R (2010) Effect of soy proteins vs soy isoflavones on lipid profile in postmenopausal women. Indian J Clin Biochem 25: 201-207. https://doi.org/10.1007/s12291-0100036-8
Kamiński M, Skonieczna-Żydecka K, Nowak J K, Stachowska E (2020) Global and local diet popularity rankings, their secular trends and seasonal variation in Google Trends data. Nutrition 79-80: 110759. https://doi.org/10.1016/j.nut.2020.110759

Kim MK, Cho SW, Park YK (2012) Long-term vegetarians have low oxidative stress, body fat, and cholesterol levels. Nutr Res Pract 6: 155-161. https://doi.org/10.4162/nrp.2012.6.2.155

Kuchta A, Lebiedzińska A, Fijałkowski M, Gałaska R, Kreft E, Totoń M, Czaja K, Kozłowska A, Ćwiklińska A, Kortas-Stempak B, Strzelecki A, Gliwińska A, Dąbkowski K, Jankowski M (2016) Impact of plant-based diet on lipid risk factors for atherosclerosis. Cardiol J 23: 141-148. https://doi.org/10.5603/CJ.a2016.0002

Lee AK, Binongo JN, Chowdhury R, Stein AD, Gazmararian JA, Vos MB, Welsh JA (2014) Consumption of less than $10 \%$ of total energy from added sugars is associated with increasing hdl in females during adolescence: A longitudinal analysis. I Am Heart Assoc 3: e000615. https://doi.org/10.1161/JAHA.113.000615

Manuelli M, Della Guardia L, Cena H (2017) Enriching diet with n-3 PUFAs to help prevent cardiovascular diseases in healthy adults: results from clinical trials. Int J Mol Sci 18: 1552. https://doi. org/10.3390/ijms18071552

Mekonen W, Haile D, Bloomer RJ (2018) Blood lipid fractions following a vegan diet in Ethiopian Society: A Study from a developing nation. Act Sci Nutr Health 2: 71-76

Melina V, Craig W, Levin S (2016) Position of the academy of nutrition and dietetics: vegetarian diets. J Acad Nutr Diet 116: 1970-1980. https://doi.org/10.1016/j.jand.2016.09.025

Mozaffarian D (2016) Dietary and policy priorities for cardiovascular disease, diabetes, and obesity: A comprehensive review. Circulation 133: 187-225. https://doi.org/10.1161/CIRCULATIONAHA.115.018585

Oussalah A, Levy J, Berthezène C, David H. Alpers DH, Guéant JL (2020) Health outcomes associated with vegetarian diets: An umbrella review of systematic reviews and meta-analyses. Clin Nutr 39: 3283-3307. https://doi.org/10.1016/j.clnu.2020.02.037

Rizzo G, Baroni L (208) Soy, soy foods and their role in vegetarian diets. Nutrients 10: 43. https://doi.org/10.3390/nu10010043

Saunders AV, Davis BC, Garg ML (2013) Omega-3 polyunsaturated fatty acids and vegetarian diets. Med J Aust 199: S22-S226. https:// doi.org/10.5694/mja11.11507

WHO Noncommunicable Diseases (NCD) Country Profiles 2018 (2020) World Health Organization. https://www.who.int/nmh/ countries/en/

Wojda A, Janczy A, Małgorzewicz S (2021) Mediterranean, vegetarian and vegan diets as practical outtakes of EAS and ACC/AHA recommendations for lowering lipid profile. Acta Biochim Pol 68: 41-48. https://doi.org/10.18388/abp.2020_5515

Wu Y, Qian Y, Pan Y, Li P, Yang J, Ye X, Xu G (2015) Association between dietary fiber intake and risk of coronary heart disease: A meta-analysis. Clin Nutr 34: 603-611. https://doi.org/10.1016/j. clnu.2014.05.009

Yokoyama Y, Levin SM, Barnard ND (2017) Association between plantbased diets and plasma lipids: a systematic review and meta-analysis. Nutr Rev 75: 683-698. https://doi.org/10.1093/nutrit/nux030

Zhou Q, Wu J, Tang J, Wang JJ, Lu ChH, Wang PX (2015) Beneficial effect of higher dietary fiber intake on plasma HDL-C and TC/HDL-C ratio among Chinese rural-to-urban migrant workers. J Environ Res Public Health 12: 4726-4738. https://doi.org/10.3390/ ijerph120504726 